\title{
Heavy Oil Characterization While Drilling
}

\section{Gabor G. Hursan, Saudi Aramco}

The recognition heavy oil and bituminous zones is a critical input for well placement in many Saudi Arabian fields. Heavy oil composition and viscosity evaluation while drilling remains a great technical challenge to this day. An additional complexity stems from permeability variations due to the complex pore system of the carbonate reservoir rock. Saudi Aramco has successfully utilized existing methods and also pioneered new technology development to acquire these critical reservoir data. This presentation begins with an overview of logging and formation testing measurements that are available while drilling for heavy oil interpretation. This includes formation testing while drilling, nuclear magnetic resonance (NMR) logs and specialized mud logging techniques as well as indirect indicators on conventional logging measurements. The rest of the presentation comprises log examples from Saudi Arabia. These examples demonstrate a comprehensive petrophysical workflow that integrates key pieces of logging and formation testing data for heavy oil evaluation. 